Revised: February 29, 2016

\title{
A Demonstration of the Incompleteness of Calculi of Inductive Inference
}

\author{
John D. Norton ${ }^{1}$ \\ Department of History and Philosophy of Science \\ Center for Philosophy of Science \\ University of Pittsburgh \\ http://www.pitt.edu/ jdnorton
}

\begin{abstract}
A complete calculus of inductive inference captures the totality of facts about inductive support within some domain of propositions as relations or theorems within the calculus. It is demonstrated that there can be no complete, non-trivial calculus of inductive inference.
\end{abstract}

\section{Introduction}

In informal accounts of inductive inference, we may say that the evidence strongly supports some scientific theory. Just how strong is "strong"? We may say that simpler or more explanatory hypotheses deserve greater inductive support. But how can we justify this when we are barely able to say just what is it to explain or to be simple?

Formal approaches to inductive inference hold the promise of a mechanical solution to such conundrums. It lies in the enticing ideal of a complete inductive calculus. In it, strengths of inductive support would be represented numerically; and their magnitudes would be fixed by the explicit rules of some calculus that render their determination a matter of mechanical computation. Then all relations of inductive support in some domain, as well as all general facts

${ }^{1}$ I am grateful for helpful discussion especially to Wayne Myrvold and to Yann BenétreauDupin, Greg Gandenberger, Paul Teller, Jim Woodward and the Fellows of the Center for Philosophy of Science, Spring Term, 2015. 
about them, would be captured fully as relations and theorems within that calculus.

Such a calculus could, for example, capture the totality of all inductive relations of support that ground our present science in a single, enormous computation. The actual computation would, of course, be prohibitively complicated. No one should expect that it could be written down in any fashion tractable to us. However its possibility in principle would be of the highest foundational importance. It would mean that all specific facts about the inductive support of some particular proposition on some particular body of evidence could be captured as relations fully derivable within the calculus; and all general facts about inductive inference would be reducible to theorems in the applicable calculus. The foundational puzzles of inductive inference would yield eventually and inexorably to the display of suitable theorems. The conundrums of philosophical analysis would have been replaced by the mechanics of theorem proving.

Call this all-embracing capacity of a quantitative calculus its "inductive completeness." What will be demonstrated here is that there can be no inductively complete calculus. The result pertains to no particular calculus of inductive inference, but to the prospects of a broad class of them to be characterized below. The class includes the probability calculus favored by the Bayesians.

The demonstration requires assumptions. The two most important are these:

First, the requirement of completeness is implemented as a requirement that the inductive logic draw only on resources within the compass of the propositions at issue. No external judgments of strengths of inductive support can be used, for they would lie outside the inductive scrutiny of the logic. Since those internal resources are just the deductive relations among the propositions, the inductive logic is required to be "deductively definable." That is, the strengths of inductive support are defined either explicitly or implicitly in terms of the deductive relations (i.e. "what deductively entails what") over the algebra of propositions at issue. An illustration of an implicit definition of this type is the defining of probability measures through Kolmogorov type axioms, supplemented with further propositions that fix the probabilities. This condition, and the symmetry theorem derived from it, are developed in Sections 3 and 4.

Second it is assumed that the logic is "asymptotically stable." This condition responds to the possibility of refining, that is, expanding, the algebra of propositions by disjunction. The proposition that there will be a solar eclipse on June 1st can be replaced by the disjunction of propositions asserting a solar eclipse on the morning of June 1st or on the afternoon of June 1st. 
The strength of support afforded to some fixed proposition by some fixed evidence may be altered by the refinement. The presumption of asymptotic stability is that this strength of support converges towards a unique limit that is the best representation of the strength of support; and that all continuing refinements eventually become inductively inert hair-splitting that does not disrupt the approach to this unique limit. This condition is developed in Section 5.

The no-go result, developed in Sections 6, is that any inductive logic satisfying these two conditions and an additional continuity condition reduces to a trivial logic in which the inductive strengths of support converge to a single value.

In barest form, the proof depends on the fact that a deductively definable logic of induction is adapted to a deductive structure that is highly symmetric, so that the inductive structure inherits all its symmetries. These symmetries render the inductive logic unable to discern that continued disjunctive refinement eventually becomes inductive hair-splitting. Instead, its inductive strengths keep responding in inconsistent ways to different refinements and fail to stabilize, unless the logic is trivial. The strategy of the demonstration is akin to the familiar use of the principle of indifference to show that there can be no neutral prior probability distribution. That strategy is amplified here greatly and applied not just to prior strengths of support, but to all strengths of support and the rules of the logics themselves.

Section 8 reviews various escapes intended to preserve the possibility of a complete calculus of inductive inference. Among them is the possibility of escape through subjective Bayesianism, where inductive inferences are embedded in a larger context of the dynamics of belief states. While the possibility of arbitrarily chosen prior beliefs breaks the symmetry central to the no-go result, the escape fails since the problems driving the no-go result reappear in a different guise. Similarly, escapes by designating preferred refinements or preferred languages amount to a failure of completeness, for inductive considerations outside the domain must determine which partitions or languages should be preferred.

The main significance of this analysis is explored in Section 7. It is that inductive logic cannot be reduced to a single mathematical calculus. Any non-trivial calculus is incomplete and a non-trivial application is possible only if one introduces additional inductive content that comes from outside the calculus and is therefore beyond the scope of its inductive scrutiny. This additional content may manifest as a need to stipulate sensible Bayesian prior probabilities through external consideration or in a failure of a novel calculus to accommodate all problems. 
These difficulties are misdiagnosed as temporary nuisances, such as the "problem of the priors," while they are really manifestations of an ineliminable foundational problem.

In a much quoted passage, De Finetti $(1938,194)$ proclaimed the reduction of inductive reasoning to probability theory:

...one must invert the roles of inductive reasoning and probability theory: it is the latter that has autonomous validity, whereas induction is the derived notion. One is thus led to conclude with Poincaré that "whenever we reason by induction we make more or less conscious use of the calculus of probabilities."

The import of the results of this paper is that this reduction of induction to probability, or to any other of a broadly defined class of calculi, cannot be achieved completely. We cannot answer the question of "what is inductive inference?" with "It is merely inference governed by such and such a calculus."

Most of the literature seeks to show what an inductive calculus can do, not what it cannot do. Thus these sorts of incompleteness results are relatively rare. For one that developed into the learning theoretic paradigm, see Putnam (1963) and its extensively developed versions in Kelly (1996, especially Ch. 13). Closer to this paper, Titelbaum $(2010,2011)$ has extended Goodmangrue like considerations to a permutation symmetry among predicates in an unquantified predicate logic. He argues that the symmetry precludes a purely syntactic relation in which hypothesis $h_{1}$ is favored over hypothesis $h_{2}$ by evidence e.

The results to be discussed extend those developed in a simpler form in Norton (2010) for a different purpose. The goal of the present paper is to give a full statement of the incompleteness and its proof, with emphasis on the technical details. Further interpretive remarks, a simplified encapsulation and discussion of the significance of the incompleteness can be found in Norton (2015).

\section{The Deductive Structure}

\subsection{Finite Boolean Algebras of Propositions}

An inductive calculus defines relations of inductive support over some set of propositions, connected by deductive relations. We shall proceed with the simple case of the 
sentential logic of arbitrarily many, but only finitely many, non-compounded propositions. This simple logic will suffice for the no-go result. (See Section 8.1 for further discussion.) That is, we consider finitely many propositions $\mathrm{A}, \mathrm{B}, \mathrm{C}, \ldots$ and the compound sentences formed from them using the operators ("not"), v (“or") and \& ("and"). This representation admits many compound propositions that are logically equivalent and thus, for our purposes, the same. For example, $\mathrm{A} \& \mathrm{~A}, \mathrm{~A}$ v $\mathrm{A}, \mathrm{A} \&(\mathrm{~B} \sim \mathrm{B})$ are all logically equivalent to $\mathrm{A}$.

The better representation is through Boolean algebras of different sizes. Their sizes are measured by the number of atoms, which is the number of logically incompatible, deductively strongest, non-contradictory propositions in the algebra. A three-atom algebra, with atoms $\mathrm{a}_{1}, \mathrm{a}_{2}$ and $\mathrm{a}_{3}$, has just 8 logically distinct propositions:

$$
\begin{aligned}
& \varnothing \text { (the contradiction) } \\
& \mathrm{a}_{1}, \mathrm{a}_{2}, \mathrm{a}_{3} \\
& \mathrm{a}_{1} \mathrm{v} \mathrm{a}_{2}, \mathrm{a}_{1} \mathrm{v} \mathrm{a}_{3}, \mathrm{a}_{2} \mathrm{v} \mathrm{a}_{3} \\
& \Omega_{3}=\mathrm{a}_{1} \mathrm{v} \mathrm{a}_{2} \mathrm{v} \mathrm{a}_{3} \text { (the universal proposition) }
\end{aligned}
$$

The set of propositions over which inductive relations will be defined will include all such algebras with finitely many atoms: $\Omega_{2}, \Omega_{3}, \Omega_{4}, \ldots$

\subsection{Symmetries of the Boolean Algebra}

The deductive structure of a Boolean algebra is just the full set of all deductive entailment relations among the propositions, such as

$$
\varnothing \text { entails } \mathrm{a}_{1} \text { entails }\left(\mathrm{a}_{1} \mathrm{v} \mathrm{a}_{2}\right) \text { entails }\left(\Omega_{3}=\mathrm{a}_{1} \mathrm{v} \mathrm{a}_{2} \mathrm{v} \mathrm{a}_{3}\right)
$$

This deductive structure is highly symmetric. For example, the two atoms $a_{1}$ and $a_{2}$ enter into deductive relations that are perfect copies of another. Relations

$$
\begin{aligned}
& a_{1} \text { entails } a_{1} v a_{2} \\
& a_{2} \text { entails } a_{2} v a_{3}
\end{aligned}
$$

are two copies of the more general entailment relation

(some atom) entails (some atom) v (some other atom)

The two differ only in the choice of labels of the atoms. The general result is that the deductive structure is preserved under arbitrary relabeling of the atom. Such relabeling includes arbitrary 
permutation of the atom labels. For more discussion of these symmetries, including pictorial representations, see Norton (2010, Section 3).

\section{Deductively Definable Logics of induction: The Formal Expression of Completeness}

\subsection{Strength of Inductive Support}

An inductive logic assigns inductive strengths "[AlB]" to pairs of propositions in a Boolean algebra, where this symbol represents the degree to which proposition A is inductively supported by proposition B. (On other possibilities, see Section 8.2.) What makes these quantities strengths is that we can say that one is stronger or weaker than another. More precisely, we assume that there is a partial order relation "s" defined over all well-defined strengths. ${ }^{2}$ That is, the relation is reflexive, antisymmetric and transitive. So that non-trivial limits are possible, the set of strengths is also assumed to be dense. ${ }^{3}$ There are two extreme values. There is a unique maximum value when $\mathrm{A}$ entails $\mathrm{B}$ and ${ }^{4} \mathrm{~B}$ entails $\mathrm{A},[\mathrm{A} \mid \mathrm{B}]=[\mathrm{A} \mid \mathrm{A}]=$ $[\Omega \mid \Omega]$. When B entails $\sim \mathrm{A},[\mathrm{A} \mid \mathrm{B}]$ has the unique minimum $[\mathrm{A} \mid \mathrm{B}]=[\varnothing \mid \mathrm{B}]=[\varnothing \mid \Omega]$.

If the rules that specify the values of these strengths are sufficiently unambiguous that computation of the strengths is mechanical, then we have a calculus of inductive inference.

Our concern is the completeness of the calculus, as described in Section 1. That is, we seek a calculus that draws only on the resources of the propositions within the algebra. These resources are restricted to the deductive relations among the propositions, that is, its deductive structure. External inductive content, that is, externally generated judgments of strengths of

${ }^{2}$ For some pairs of propositions, strengths may not be defined. For example $\mathrm{P}(\mathrm{A} \mid \varnothing)$ is undefined in probability theory.

3 That is, for any strengths $\mathrm{x}$ and $\mathrm{y}$ such that $\mathrm{x}<\mathrm{y}$, there exists a $\mathrm{z}$ such that $\mathrm{x}<\mathrm{z}<\mathrm{y}$. The relation $\mathrm{x}<\mathrm{y}$ is defined as $\mathrm{x} \leq \mathrm{y}$ but not $\mathrm{x}=\mathrm{y}$.

${ }^{4}$ For a probabilistic logic, the condition that A entails B is superfluous. However it is required by other inductive logics, such as the "specific conditioning" logic of Norton $(2010, \S 11.2 ; 2015$, Part II). 
inductive support, are excluded. Hence the condition of completeness is implemented by requiring that the rules specifying the strengths of support employ only the deductive structure of the algebra of propositions. Such an inductive logic is "deductively definable." There are two modes of definition: explicit and implicit. 5

The two types of definition below pertain to some fixed set of $m$ propositions $\left\{A_{1}, A_{2}\right.$, ..., $\left.\mathrm{A}_{\mathrm{m}}\right\}$. This set is intended to be very large. It might consist, for example, of all the hypotheses of a science along with the propositions describing the evidence for the science. The set is embedded in a Boolean algebra of propositions. It is fixed in the sense that the set will remain unaltered while we enlarge the Boolean algebra by disjunctive refinements introduced in Section 5.1 .

\subsection{Explicit Definition}

More traditional accounts of inductive logic tend to support explicit definition. The venerable enumerative induction has the universal affirmation "A: Every S is P." inductively supported by the particular affirmation "I: Some S is P." The relation of I supporting A is defined explicitly in terms of the deductive relations between the universal A and the particular I. Hempel's (1965) satisfaction criterion of confirmation allows that $\mathrm{P}(\mathrm{a})$ \& $\mathrm{P}(\mathrm{b})$ confirms the universally quantified (x) $\mathrm{P}(\mathrm{x})$, since $\mathrm{P}(\mathrm{a})$ \& $\mathrm{P}(\mathrm{b})$ is what Hempel calls the development of (x) $\mathrm{P}(\mathrm{x})$ with respect to the domain $\{\mathrm{a}, \mathrm{b}\}$. Once again, the confirmation relation is defined explicitly only in terms of deductive relations.

In comparison to the propositional logic, these last two examples employ respectively a weaker syllogistic logic and a stronger predicate logic. Simple hypothetico-deductive confirmation can be explicitly defined within a propositional logic:

E hypothetico-deductively confirms $\mathrm{H}$ just in case $\mathrm{H}$ deductively entails $\mathrm{E}$. or more elaborately

E hypothetico-deductively confirms $\mathrm{H}$ with respect to auxiliaries B just in case $\mathrm{H} \& \mathrm{~B}$ deductively entails $\mathrm{E}$.

\footnotetext{
5 This paper gives a more precise definition than provided in Norton $(2010, \S 5)$ and extends it to include implicit definitions.
} 
Simple hypothetico-deductive confirmation is generally regarded as too permissive. It is usually augmented with further conditions that may require that $\mathrm{H}$ be, in some sense, simple; or that $\mathrm{H}$ not just deductively entail $\mathrm{E}$, but explain it. It may happen that these extra conditions can be made explicit enough for logical formulation; and that the algebra of propositions can be suitably expanded to express them. ${ }^{6}$ Then the augmented confirmation relation will still be deductively definable.

This suggests the following formulation for the explicit definition: Explicit definition of $\left[A_{i} l A_{k}\right]$ for $i, k=1, \ldots, m$ $\left[\mathrm{A}_{\mathrm{i}} \mid \mathrm{A}_{\mathrm{k}}\right]=$ formula that mentions only the deductive relations among some set ${ }^{7}$ of propositions of the algebra $\left\{\mathrm{A}_{1}, \mathrm{~A}_{2}, \ldots, \mathrm{A}_{\mathrm{m}}\right\}$ and their deductive relations to the atoms of the algebra $\Omega$.

Since each proposition is formed as a disjunction of atoms, inductive strengths can be defined explicitly as a function of the number of atoms in the disjunction. (The symmetry theorem below will show that all explicit definitions reduce to such a function.) The simplest example is the classical definition of probability:

$$
[\mathrm{Al} \mathrm{B}]=\# \mathrm{~A} \& \mathrm{~B} / \# \mathrm{~B}
$$

where \#A\&B is the number of atoms in the disjunction of atoms equivalent to $A \& B$; and similarly for \#B.

${ }^{6}$ Otherwise, we may not have a relation of support precise enough to be captured by a calculus. The defining of the relation may require further facts about explanation and simplicity that cannot be expressed within the algebra of proposition. Then the inductive logic is revealed at the start as incomplete, for these further facts lie beyond the inductive scrutiny of the inductive logic. ${ }^{7}$ Natural explicit definitions typically employ only the deductive relations among $A_{i}$ and $A_{k}$ and the atoms. The m-membered set of propositions is included for continuity of exposition with the implicit definition. Note that a set is not ordered, so that the numbering of the propositions in the set is arbitrary. 


\subsection{Implicit Definition}

In more recent work, the degrees of support are introduced implicitly by the requirement that they satisfy some system of axioms. This suggests the following formulation for implicit definition.

Implicit definition of $\left[A_{i} l A_{k}\right]$ for $i, k=1, \ldots, m$

Sentences that mention only the strengths $\left[A_{i} l A_{k}\right]$, for $i, k=1, \ldots, m$, for propositions of the algebra in the set $\left\{A_{1}, \ldots, A_{m}\right\}$, the deductive relations among $A_{1}, \ldots, A_{m}$ and their deductive relations to the atoms of the algebra $\Omega$. The sentences uniquely fix the strengths. Merely requiring conformity to commonly used axiom systems, such as that for the probability calculus, is insufficient to specify the strengths uniquely. So, typically, additional sentences will be required in the implicit definition in order to fix the strengths. These sentences will likely be specific to the particular inductive problem under investigation.

For example, an implicit definition of a probabilistic system of real-valued strengths of support might include, along with others, the following sentences:

For all $A_{i}, A_{j}$ and $A_{k}$ where $A_{i} \& A_{j}=\varnothing$,

$$
\left[\mathrm{A}_{\mathrm{i}} \mathrm{vA} \mathrm{A} \mid \mathrm{A}_{\mathrm{k}}\right]=\left[\mathrm{A}_{\mathrm{i}} \mid \mathrm{A}_{\mathrm{k}}\right]+\left[\mathrm{A}_{\mathrm{j}} \mathrm{l} \mathrm{A}_{\mathrm{k}}\right] .
$$

For all $A_{i}, A_{j}$ and $A_{k}$, where neither $A_{i}$ nor $A_{j}$ are $\varnothing$,

$$
\left[A_{i} \& A_{j} \mid A_{k}\right]=\left[A_{i} \mid A_{j} \& A_{k}\right] x\left[A_{j} \mid A_{k}\right] .
$$

For all $A_{i}$, and $A_{k}$, if propositions $A_{i} \& A_{k}=\varnothing$ and there are no $A_{r}$ and $A_{s}$, where $i \neq r$ and $s \neq k$, such that $A_{r}$ entails $A_{i}$ and $A_{s}$ entails $A_{k}$ then $\left[\mathrm{A}_{\mathrm{i}} \mid \Omega\right]=\left[\mathrm{A}_{\mathrm{k}} \mid \Omega\right]$.

The first two sentences are familiar axioms of conditional probability and the third is a specific condition one might choose to assist in securing unique values of the inductive strengths. This particular one is an attempt to introduce a uniform prior probability.

The analysis that follows will depend upon a key common feature of both explicit and implicit definitions: 
For a deductively definable logic of induction, for some set $\left\{A_{1}, \ldots, A_{m}\right\}$ of propositions in the algebra, the pairwise inductive strengths $\left[A_{i} l A_{k}\right]$ (for $i, k=1$,

$\ldots, \mathrm{m})$ are determined uniquely by the deductive relations among the propositions $\mathrm{A}_{1}, \ldots, \mathrm{A}_{\mathrm{m}}$ and their deductive relations with the atoms of the algebra.

\section{The Symmetry Theorem}

\subsection{An Illustration}

Since the deductive structure is highly symmetric, the requirement of deductive definability places a powerful restriction on the strengths of inductive support: the inductive structure must inherit all the symmetries of the deductive structure.

Take the simple example of the two strengths

$$
[A \mid B]=\left[a_{1} \mid a_{1} \vee a_{2} \vee a_{3}\right] \text { and }\left[A^{\prime} \mid B^{\prime}\right]=\left[a_{2} \mid a_{1} \vee a_{2} \vee a_{3}\right]
$$

The deductive relations among $\mathrm{A}=\mathrm{a}_{1}$ and $\mathrm{B}=\mathrm{a}_{1} \mathrm{va}_{2} \mathrm{va}_{3}$ are the same as the deductive relations among $\mathrm{A}^{\prime}=\mathrm{a}_{2}$ and $\mathrm{B}=\mathrm{B}^{\prime}=\mathrm{a}_{1} \mathrm{va}_{2} \mathrm{va}_{3}$. In each case we have the support a single atom accrues from a disjunction of three atoms that include the atom. The deductive relations between the atom and the disjunction are the same in each case. Hence, if we require that the strengths are deductively definable in terms of these particular deductive relations alone, then the strengths must be the same.

We can arrive at the same result by a more formal procedure. If we merely swap the labels " $a_{1}$ " and " $a_{2}$ " then $[A \mid B]=\left[a_{1} \mid a_{1} \vee a_{2} \vee a_{3}\right]$ is mapped to $\left[a_{2} \mid a_{2} \mathrm{va}_{1} \mathrm{va}_{3}\right]$, which is $\left[a_{2} \mid a_{1} v_{2} a_{3}\right]=\left[A^{\prime} \mid B^{\prime}\right]$ since $a_{2} \vee a_{1} v a_{3}=a_{1} v_{2} a_{2} a_{3}$. Since a relabeling of the atoms preserves deductive relations, the deductive relations between the two propositions in each of the strengths is the same. We have

$$
[\mathrm{A} \mid \mathrm{B}]=\left[\mathrm{a}_{1} \mid \mathrm{a}_{1} \mathrm{va}_{2} \mathrm{va}_{3}\right]=\left[\mathrm{a}_{2} \mid \mathrm{a}_{1} \mathrm{va}_{2} \mathrm{va}_{3}\right]=\left[\mathrm{A}^{\prime} \mid \mathrm{B}^{\prime}\right]
$$

\subsection{The General Case}

We can now apply this same method to the general case. For the propositions $\left\{A_{1}, \ldots\right.$, $\left.A_{m}\right\}$ of the explicit or implicit definition above, the inductive strength $\left[A_{1} \mid A_{2}\right]$ is fixed merely by 
the atom counts of the $2 \mathrm{~m}$ conjunctions of $\mathrm{A}_{1}, \ldots, \mathrm{A}_{\mathrm{m}}$ and their negations. This fact can be expressed compactly as

Symmetry Theorem. If the inductive strength $\left[\mathrm{A}_{1} \mid \mathrm{A}_{2}\right]$ is deductively definable, either explicitly or implicitly, then there exists a function $f$ of $2^{\mathrm{m}}$ integers such that

$$
\left[\mathrm{A}_{1} \mid \mathrm{A}_{2}\right]=\mathrm{f}\left(\# \mathrm{~A}_{1} \& \mathrm{~A}_{2} \& \ldots \& \mathrm{~A}_{\mathrm{m}-1} \& \mathrm{~A}_{\mathrm{m}}, \ldots, \# \sim \mathrm{A}_{1} \& \sim \mathrm{A}_{2} \& \ldots \& \sim \mathrm{A}_{\mathrm{m}-1} \& \sim \mathrm{A}_{\mathrm{m}}\right)
$$

where \#(proposition) denotes the number of atoms in the disjunction that forms the propositions. ${ }^{8}$ See Appendix A for the proof. I am calling an inductive logic based on a strength $\left[\mathrm{A}_{1} \mid \mathrm{A}_{2}\right]$ a calculus if there is an explicit rule for mechanically computing the strengths. This function $\mathrm{f}$ provides that rule. Each distinct inductive calculus is defined by a distinct function $\mathrm{f}$. Some of many possibilities are explored in Norton (2010). The most familiar is the classical definition of probability mentioned earlier, for which

$$
[\mathrm{A} \mid \mathrm{B}]=\# \mathrm{~A} \& \mathrm{~B} /(\# \mathrm{~A} \& \mathrm{~B}+\# \sim \mathrm{A} \& \mathrm{~B})=\# \mathrm{~A} \& \mathrm{~B} / \# \mathrm{~B}
$$

Another possibility among many is a scale-free, "specific conditioning" logic for which

$$
[\mathrm{AlB}]=(\# \mathrm{~A} \& \mathrm{~B} / \# \mathrm{~B}) .(\# \mathrm{~A} \& \mathrm{~B} / \# \mathrm{~A})
$$

In this logic, the support from B for proposition A is reduced if a disjunctive part of $A$ contradicts B. Conditional probability does not include such a penalty.

\section{Asymptotic Stability}

\subsection{Illustrations}

A Boolean algebra $\Omega_{\mathrm{N}}$ of size $\mathrm{N}$ with atoms $\mathrm{a}_{1}, \mathrm{a}_{2}, \ldots, \mathrm{a}_{\mathrm{N}}$ can be enlarged by replacing individual atoms by disjunctions of new atoms in a process here called "disjunctive refinement." For example, we might introduce two new atoms, $b_{1}$ and $b_{2}$ through $a_{1}=b_{1} v b_{2}$ to form a new algebra $\Omega_{\mathrm{N}+1}$ of size $\mathrm{N}+1$ with atoms $\mathrm{b}_{1}, \mathrm{~b}_{2}, \mathrm{a}_{2}, \ldots, \mathrm{a}_{\mathrm{N}}$.

Sometimes the added expressive power of disjunctive refinement is not needed. Take the simple example of a die throw. The usual probabilistic analysis is captured by a six atom algebra with the equiprobable atoms "one," "two," ..., "six" representing the outcomes. The algebra

\footnotetext{
${ }^{8}$ For example, if proposition $\mathrm{A}$ is a disjunction of three atoms $\mathrm{a}_{1} \mathrm{v} \mathrm{a}_{2} \mathrm{v} \mathrm{a}_{3}$, then $\# \mathrm{~A}=3$.
} 
could be refined by adding information on whether the thrown die landed on the left hand side of the table ("left") or the right ("right"). We could form a seven-atom algebra with atoms

one \& left, one \& right, two, ..., six

However, since the outcome of a well-thrown die toss is independent of the side of the table on which the die lands, the disjunctive refinement will not affect the probabilities of the original six outcomes.

In other cases, the added expressive power will be important. Consider a dart thrown onto a square dartboard. We are interested in the probability that the dart lands in the largest circle that can be fitted into the square. Assuming a uniform distribution of probability over area, the probability of the dart landing inside the circle ("inside") is $\mathrm{P}$ (inside) $=\pi / 4=0.785398 \ldots$ If we assume equiprobable atoms, we might approximate this by a ten atom algebra in which the two outcomes are represented by

$$
\text { inside }=\mathrm{a}_{1} \mathrm{va}_{2} \mathrm{v} \ldots \mathrm{v} \mathrm{a}_{8} \quad \text { outside }=\mathrm{a}_{9} \mathrm{va}_{10}
$$

Hence $\mathrm{P}($ inside $)=0.8$. We can come closer to the correct value by a tenfold refinement of each atom so that we have an algebra of one hundred atoms. Then we set

$$
\text { inside }=\mathrm{a}_{1} \mathrm{va}_{2} \mathrm{v} \ldots \mathrm{v} \mathrm{a}_{79} \quad \text { outside }=\mathrm{a}_{80} \mathrm{v} \mathrm{a}_{81} \mathrm{v} \ldots \mathrm{v} \mathrm{a}_{100}
$$

and we have $\mathrm{P}($ inside $)=0.79$. By continuing with these disjunctive refinements we can come arbitrarily close to the sought probability of $\mathrm{P}($ inside $)=\pi / 4$.

\subsection{The General Condition}

In general, in a deductively definable logic, disjunctive refinements will lead to adjustments of the inductive strengths of support, for both explicit and implicit definitions include mention of deductive relations to the atoms; and disjunctive refinement alters the atoms. These adjustments can be important, as the dartboard example shows. The supposition of asymptotic stability is that eventually, under repeated disjunctive refinements, these adjustments of inductive strengths either stop completely or the strengths converge toward fixed values. These last values are the representation of the true strengths; the earlier values appearing in the process of convergence are merely approximations to them. We have:

Asymptotic Stability Under Disjunctive Refinement

For some fixed set of propositions $\left\{A_{1}, \ldots, A_{m}\right\}$ of the explicit or implicit definition of a deductively definable logic of induction, for each strength $\left[A_{i} \mid A_{k}\right]$, 
$\mathrm{i}, \mathrm{k}=1, \ldots, \mathrm{m}$, there exists a limiting value, possibly unique to that strength, $\left[\mathrm{A}_{\mathrm{i}} \mid \mathrm{A}_{\mathrm{k}}\right]_{\text {lim }}$ to which the strength converges under all possible disjunctive refinements of the algebra. In taking the limit, it is assumed that disjunctive refinement of each proposition can be continued indefinitely ${ }^{9}$

The sense of convergence is the obvious one: For any strengths $V, V^{\prime}$, where $V<\left[A_{i} \mid A_{k}\right]_{\lim }<$ $\mathrm{V}^{\prime}$, it is possible to carry out a disjunctive refinement on the algebra such that, in the refined algebra, $\left[A_{i} \mid A_{k}\right]$ satisfies $V<\left[A_{i} \mid A_{k}\right]<V$, and remains so for all subsequent disjunctive refinements.

Informally, disjunctive refinements can enrich the expressive power of the algebra of propositions in ways essential to the inductive problem. However eventually all further disjunctive refinement becomes inductive hair-splitting that no longer affects the inductive strengths considered originally.

Why require asymptotic stability? Without it, there is no assurance that the strengths of inductive support defined among the propositions $A_{1}, \ldots, A_{m}$ have unique values. For the set may be embedded in many algebras of propositions and, in general in a deductively definable logic of induction, each embedding will yield a different set of strengths. It would be too strong to require that all embeddings yield the same strengths. A weaker way to deal with the problem is to require merely that the strengths stabilize when the algebras become very large.

\section{The No-Go Result}

While deductive definability and asymptotic stability are individually desirable properties, the no-go result shows that their conjunction collapses all the logics to a trivial inductive logic with just one limiting strength of support. Section 6.1 illustrates the proof method. The result is given in Section 6.2 and the proof is provided in Appendix B.

\footnotetext{
${ }^{9}$ For propositions concerning continua, such as the case of the dartboard, the possibility of this indefinite continuation is automatic. For propositions concerning discrete properties, the indefinite continuation can be effected by artifices such as conjunctions with propositions concerning continua.
} 


\subsection{Illustration: The Principle of Indifference.}

The idea behind the result is that a deductively definable logic of induction does not have the internal resources to know when disjunctive refinement should no longer alter the strengths of support. Take the case of two atoms, $\mathrm{a}_{1}$ and $\mathrm{a}_{2}$. From the symmetry theorem we have:

$$
\left[a_{1} \mid a_{1} v a_{2}\right]=\left[a_{2} \mid a_{1} v a_{2}\right]
$$

for each strength is schematically "[1 atom I 2 atoms]".

We now confuse the logic by malicious refinements. First, we refine the atom $\mathrm{a}_{1}$ into 99 disjunctive parts:

$$
a_{1}=b_{1} v b_{2} \vee \ldots v b_{99}
$$

The two strengths become:

$$
\begin{aligned}
{\left[a_{1} \mid a_{1} v a_{2}\right]=} & {\left[b_{1} v b_{2} v \ldots v b_{99} \mid b_{1} v b_{2} v \ldots b_{99} v a_{2}\right] } \\
& \text { vs }\left[a_{2} \mid b_{1} v b_{2} v \ldots v b_{99} v a_{2}\right]=\left[a_{2} \mid a_{1} v a_{2}\right]
\end{aligned}
$$

That is, schematically, we have:

$$
\text { "[99 atoms I } 100 \text { atoms]" vs "[1 atom I } 100 \text { atoms]" }
$$

In a generic logic, we would expect this change in atoms counts to alter the strengths, most likely to

$$
\left[a_{1} \mid a_{1} v a_{2}\right]>\left[a_{2} \mid a_{1} v a_{2}\right]
$$

What makes the refinement malicious is that, instead of refining $a_{1}$, we could refine $a_{2}$ in exactly the same way

$$
\mathrm{a}_{2}=\mathrm{c}_{1} \mathrm{v} \mathrm{c}_{2} \mathrm{v} \ldots \mathrm{v} \mathrm{c}_{99}
$$

Then by analogous reasoning we would end up with

$$
\left[a_{1} \mid a_{1} v a_{2}\right]<\left[a_{2} \mid a_{1} v a_{2}\right]
$$

This contradicts the outcome of the first refinement.

An inductive logic can protect itself from malicious refinements by flattening all strengths. For example, strengths that are schematically "[99 atoms I 100 atoms]" and "[1 atom I 100 atoms]" would be set equal. But that is to trivialize the inductive logic.

The general no-go result below shows that this trivialization is unavoidable in deductively definable logics of induction. It shows that asymptotic stability requires the strengths of inductive support to converge under repeated disjunctive refinement to a single value. 
This illustration is an amplified version of a familiar problem. The "principle of indifference" is an unassailable truism of evidence that greatly troubles probabilistic logics. Keynes $(1921,41)$ named the principle and gave an illustration (p.44) of how its use causes trouble. After antipodean relabeling of Keynes' place names, we suppose that our most refined possibilities for an Australasian is indifferently that the antipodean is a non-New Zealander or a New Zealander, The principle of indifference requires that we assign equal probability of $1 / 2$ to each. If we disjunctively refine the possibilities so that a New Zealander is replaced by the logically equivalent (North Island New Zealander or South Island New Zealander), then we have three most refined possibilities over which we are indifferent:

a non-New Zealander or a North Island New Zealander or a South Island New Zealander The principle of indifference now requires that we assign equal probabilities of $1 / 3$ to each of these; and this new assignment contradicts the old probability assignment. ${ }^{10}$

\subsection{The Result11}

The following is proved in Appendix B:

No-Go.

For a set of propositions $\left\{A_{1}, \ldots, A_{m}\right\}$ defined on finite Boolean algebras and for an inductive logic that is:

(i) deductively definable in the sense of Section 3 ,

(ii) asymptotically stable in the sense of Section 5 , and

(iii) continuous in the sense of Section B.3,

10 Norton (2008) argues that the principle of indifference is an evidential truism and that a proper accommodation of it requires a non-probabilistic logic in which all these outcomes are assigned a single ignorance value "I."

11 This result was first presented in simpler form in Norton $(2010, \S 6)$. The present version extends the notion of explicit definability, introduces implicit definability, introduces the notion of classes of deductive structures, introduces a notion of continuity and employs it. The older development erred in failing to recognize the need for a continuity condition. 
all the well-defined 12 inductive strengths $\left[\mathrm{A}_{\mathrm{i}} \mid \mathrm{A}_{\mathrm{k}}\right]$, for $\mathrm{i}, \mathrm{k}=1, \ldots, \mathrm{m}$, converge under disjunctive refinement to a single strength. This includes the maximum strength $[\Omega \mid \Omega]$ and the minimum strength $[\varnothing \mid \Omega]$.

That is, there is no inductive logic satisfying (i)-(iii) other than the trivial logic with one limiting inductive strength. Since deductive definability is a formal expression of completeness, the no-go result asserts that there is no non-trivial, asymptotically stable, continuous logic of induction that is complete.

The requirement of continuity can be dropped. The no-go result reverts to the less elegant result that all strengths in each of the classes of deductive structures defined in Appendix B.1 converge to a single value unique to that class. This result still trivializes the inductive logic.

To get a sense of the import of this no-go result, note that the set of propositions $\left\{\mathrm{A}_{1}, \ldots\right.$, $\left.\mathrm{A}_{\mathrm{m}}\right\}$ must be finite, but can be arbitrarily large. Let it be the totality of propositions in science, including all the propositions expressing evidence in science. The no-go result tells us that no inductive logic satisfying its conditions (i)-(iii) can affirm whether the propositions of science are individually well supported by the totality of evidence.

\section{Incompleteness}

The pessimistic conclusion of the last paragraph is far from the full story. There are successful calculi of inductive inference that have supplied numerous important insights into inductive inference. These applications of the calculi, however, have never been complete. Their application has always been supplemented by external inductive content, sometimes explicitly and more often tacitly. The no-go result shows that this supplement is necessary.

By external inductive content, I mean the introduction of inductive strengths of support that are not fixed by the deductive structure through the definitions of the inductive logic. In real examples, these strengths are grounded in judgments made outside the particular application at issue. The strengths appear within the application itself as stipulations.

12 This allows for the possibility of a logic leaving certain strengths undefined, such as $\mathrm{P}(\mathrm{A} / \varnothing)$ in the probability calculus. 
Here are two examples to illustrate it. The most natural supplement is to specify on external grounds that the atoms of some chosen algebra $a_{1}, a_{2}, \ldots, a_{N}$ are preferred in the sense that the strengths are to be distributed uniformly over them, so that $\left[a_{1} \mid \Omega\right]=\ldots=\left[a_{N} \mid \Omega\right]$, but that no such requirement is placed on the atoms that may result from a refinement of this algebra. For example, Norton $(2010, \S 7)$ introduces inductively preferred refinements ("partitions") to enable the definition of non-trivial logics of inductions. Their stipulation contradicts deductive definability, since the strengths can no longer be defined solely in terms of the deductive structure but must recognize the externally stipulated, preferred refinement. Hence the no-go result is blocked.

A second example is the introduction of "Solomonoff priors" into a probabilistic analysis (Solomonoff, 1964). These prior probabilities assign higher probability to algorithmically less complex hypotheses. The external inductive content derives from the assumption that the world is such that inductive preference ought to be given to such hypotheses. These higher probabilities appear within the ensuing probabilistic calculations as a stipulation.

In these and other cases, the needed, external inductive content should itself have proper inductive support. If that support is provided by computations that formally use the same inductive calculus, one might try to merge those computations into the original computation. One might hope that the resulting combined computation is inductively complete. However it cannot be, since the no-go result would then apply to the combined computation. At best, we can have separate analyses: the original computation and a distinct one that grounds the external inductive content that supplements the original computation.

The need for the results of one computation to be carried over to a second underscores that the one calculus cannot be all there is to inductive inference. We also need a separate vehicle to transport the results between the computations. That is, on the model of incompleteness in arithmetic, we need a meta-theory to govern the transporting. An example of such a metaprinciple is Lewis' "principal principle" adapted to the present context. According to it the strength of support for some outcome is matched to the physical chance of its occurrence, where the physical chance is learned inductively in a different domain. 


\section{Unsuccessful Escapes}

Here are some ways one might try to escape the no-go result.

\subsection{Enriching the Deductive Logic}

Might the no-go result be escaped if we use a richer logic? It is unlikely to help. Two properties must be present in the deductive logic if a no-go result of this type is to be derived. The logic must admit a deductive structure rich enough in symmetries for a version of the symmetry theorem to be derived; and the logic must admit disjunctive refinements. These features will remain in familiar enrichments. However the complications introduced by the enrichments may make the no-go result harder to see and more complicated to prove.

For example, if we move to infinite Boolean algebras, the deductive structure remains highly symmetric and the disjunctive refinements are still possible in the same way. However we now have added complications. If our logic seeks to impose a uniform additive measure over the atoms, there is no normalizable measure that is uniform over a countable infinity of atoms. We may seek to avoid these problems by introducing further complications, such as a denial of countable additivity. The analysis becomes harder, but the no-go result will persist.

If we move to a predicate logic, the circumstances are much the same. The symmetries remain and disjunctive refinements are still possible. We might, for example, disjunctively refine the logic's predicates. A predicate $A_{1}(x)$ might be refined to $B_{1}(x) \vee \ldots v B_{N}(x)$.

\subsection{Enrich the Inductive Logic}

The inductive logic of this paper takes a two-place function $[\mathrm{HIE}]$ as its basic quantity. What of a logic that employs a three-place function: $[\mathrm{HIE}, \mathrm{B}]$, which we might interpret as the strength of inductive support of hypothesis $\mathrm{H}$ from evidence $\mathrm{E}$ with respect to background B? Or what of a four-place comparative function $\left[\mathrm{H}_{1}, \mathrm{H}_{2} \mathrm{E}, \mathrm{B}\right]$, which we might interpret as the relative inductive support of hypothesis $\mathrm{H}_{1}$ compared to hypothesis $\mathrm{H}_{2}$ from evidence $\mathrm{E}$ with respect to background B?

A review of the argumentation leading up to the no-go results shows that it makes little use of the restriction to a two-place function and that extended versions employing higher order functions could be developed with little change. 
The simplest case would be when the higher order functions are defined as functions of the two place function, such as $[\mathrm{HIE}, \mathrm{B}]=\mathrm{F}([\mathrm{HIE} \& \mathrm{~B}],[\mathrm{HIB}])$ for some function $\mathrm{F}$. Then, the nogo result extends automatically to the higher order functions.

\subsection{Preferred Refinements and Preferred Languages}

We might try to escape the no-go result by disallowing arbitrary disjunctive refinements. We would then stipulate which refinement gives the algebra of propositions that must be used and how the propositions $\mathrm{A}_{1}, \ldots, \mathrm{A}_{\mathrm{m}}$ are to be embedded in it. This algebra becomes our preferred language. This stipulation amounts to a violation of completeness. For it amounts to the designation of a preferred refinement, as described in Section 7, and this in turn amounts to the introduction of strengths of inductive support on the basis of external, inductive considerations. For example, we might stipulate an unrefinable six-atom algebra for a die problem. If the logic is deductively definable, this amounts to assuming equal support for each of the die's six faces.

\subsection{The Subjective Turn}

An initially promising approach is to follow the subjective Bayesians and conceive of conditional probabilities as degrees of belief, with the prior probabilities freely chosen, subjective opinions. This free choice of the prior probabilities breaks the symmetry essential to the derivation of the symmetry theorem of Section 4, thereby blocking the no-go result that derives from it.

This stratagem delays the problem but does not avoid it. Since the probabilities of the system include subjective opinion, there is no longer a direct connection to evidential warrant. The hope is that, as conditionalization on evidence proceeds, the stain of subjective opinion will "wash out" leaving behind pristine inductive support. This hope has been formalized in limit theorems that show convergence to the truth of initially divergent prior probabilities under continuing conditionalization on evidence, such as in Savage (1954, pp. 46-50).

The difficulty with these theorems is that there is a competing indelibility theorem. Take the ratio form of Bayes' theorem

$$
\frac{P\left(H_{1} \mid E\right)}{P\left(H_{2} \mid E\right)}=\frac{P\left(E \mid H_{1}\right)}{P\left(E \mid H_{2}\right)} \cdot \frac{P\left(H_{1}\right)}{P\left(H_{2}\right)}
$$

Assume that the growing evidence favors $\mathrm{H}_{1}$ in the limit. Then, as the evidence accumulates and 
the limit is approached, the likelihood ratio $\mathrm{P}\left(\mathrm{ElH}_{1}\right) / \mathrm{P}\left(\mathrm{ElH}_{2}\right)$ will continue to grow larger. We would expect a correspondingly large posterior ratio $\mathrm{P}\left(\mathrm{H}_{1} \mid \mathrm{E}\right) / \mathrm{P}\left(\mathrm{H}_{2} \mid \mathrm{E}\right)$ to reflect that stronger support of $\mathrm{H}_{1}$. However, since the ratio of priors $\mathrm{P}\left(\mathrm{H}_{1}\right) / \mathrm{P}\left(\mathrm{H}_{2}\right)$ is freely chosen, at any definite stage of the conditionalization, there will be choices unfavorable enough to $\mathrm{H}_{1}$ to force the ratio of posteriors to be arbitrarily small and thus to indicate lack of support of $\mathrm{H}_{1}$. For any fixed evidence, there will be a prior probability ratio that can return any nominated ratio of posteriors.

The difficulty is similar to that introduced by arbitrary disjunctive refinements for a logic of induction. Different choices of disjunctive refinement can drive the strengths of support in wildly different directions. The no-go result escapes the difficulty by reducing the inductive logic to a trivial logic. We could instead continue to use non-trivial logics of induction, however, if we introduce external inductive content and only permit disjunctive refinements that conform with it.

A quite similar escape is available to the subjective Bayesians. Instead of allowing arbitrary prior probabilities, they can require that the prior probabilities be chosen in some way that is reasonable or responsible, such as articulated by Shimony’s $(1970, \S 3)$ "tempered" personalism. Presumably what makes these constraints reasonable is that they are responsive to further, external evidence. In both cases, the collapse into arbitrariness is avoided by external inductive content. In both cases, the price paid in avoiding the collapse is that the logics are not complete.

In a related approach, confirmation measures are introduced as functions of the subjective probabilities, such as in Fitelson (1999). For example the distance measure d $(\mathrm{H}, \mathrm{ElB})=$ $\mathrm{P}(\mathrm{HIE} \& \mathrm{~B})-\mathrm{P}(\mathrm{HIB})$ gives one measure of the degree of confirmation of hypothesis $\mathrm{H}$ from evidence E. These measures also fail to escape the no-go result. There are two modes of failure.

If the measure retains a dependency on the prior probabilities, as does the distance measure $\mathrm{d}(\mathrm{H}, \mathrm{ElB})$ above, then it does not supply the sought strength of inductive support. It is an amalgam of strength of support and prior opinion.

If the measure is not dependent on the prior probability or some other external inductive content, then we have a strength of support that conforms with the framework of this paper. Therefore, some form of the no-go result will apply to it and its application cannot be both nontrivial and complete. The best candidate for a measure independent of the prior probabilities is 
the likelihood ratio $\mathrm{L}\left(\mathrm{H}_{1}, \mathrm{H}_{2} \mid \mathrm{E}\right)=\mathrm{P}\left(\mathrm{ElH}_{1}\right) / \mathrm{P}\left(\mathrm{ElH}_{2}\right)$, since likelihoods are often introduced objectively, such as through physical chances. Recent work by Gandenberger (manuscript) shows that this measure is beset by difficulties structurally quite similar to those raised by the principle of indifference for prior probabilities and thus close to the general difficulties raised in this paper.

\section{Conclusions}

What has been established here is that inductive inference cannot be captured in a complete inductive calculus.

The local import of this result is small. It does not preclude the local application of nontrivial calculi of induction. However their successful applications will always require a supplement of further inductive content, external to the calculus and beyond the reach of its inductive scrutiny. The need for this supplement has been routinely misdiagnosed as a minor failing of a particular implementation. It is really an unavoidable outcome of incompleteness.

The global import is greater. It concerns the nature of inductive inference itself. We cannot conceive of it formally, as merely inference conforming to such and such a quantitative calculus, probabilistic or otherwise. For no such calculus can be complete. We need a different conception of the nature of inductive inference. Such a conception is supplied by the material theory of induction (Norton, 2003). Inductive inferences are not warranted by conformity with universally applicable formal schema. They are warranted by facts.

\section{Appendices}

\section{A. Proof of the Symmetry Theorem}

Consider any two of the propositions $\left\{\mathrm{A}_{1}, \ldots, \mathrm{A}_{\mathrm{m}}\right\}$ of the explicit or implicit definition. Permute their labels so that the chosen two become $A_{1}$ and $A_{2}$. Now pick any other two and relabel all the propositions in the set so that they become $\mathrm{B}_{1}$ and $\mathrm{B}_{2}$ of $\mathrm{B}_{1}, \ldots, \mathrm{B}_{\mathrm{m}}$. In a deductively definable logic, the two inductive strengths $\left[\mathrm{A}_{1} \mid \mathrm{A}_{2}\right]$ and $\left[\mathrm{B}_{1} \mid \mathrm{B}_{2}\right]$ will be equal if the deductive relations among $A_{1}$ and $A_{2}$ and the remaining propositions $A_{3}, \ldots, A_{m}$ and with the 
algebra's atoms are the same as those among $\mathrm{B}_{1}$ and $\mathrm{B}_{2}$ and the remaining propositions $\mathrm{B}_{3}, \ldots$, $\mathrm{B}_{\mathrm{m}}$ and with the algebra's atoms. This sameness of deductive relations will obtain just in case there is a permutation of the labels of the atoms so that each of the $A_{1}, \ldots, A_{m}$ become the same disjunction of relabeled atoms as the $\mathrm{B}_{1}, \ldots, \mathrm{B}_{\mathrm{m}}$ are of the originally labeled atoms.

Finally, this permutation of the atom labels is possible just in case the following $2^{\mathrm{m}}$ atom count equalities obtain:

$$
\begin{aligned}
& \# \mathrm{~A}_{1} \& \mathrm{~A}_{2} \& \ldots \& \mathrm{~A}_{\mathrm{m}-1} \& \mathrm{~A}_{\mathrm{m}}=\# \mathrm{~B}_{1} \& \mathrm{~B}_{2} \& \ldots \& \mathrm{~B}_{\mathrm{m}-1} \& \mathrm{~B}_{\mathrm{m}} \\
& \# \sim \mathrm{A}_{1} \& \mathrm{~A}_{2} \& \ldots \& \mathrm{~A}_{\mathrm{m}-1} \& \mathrm{~A}_{\mathrm{m}}=\# \sim \mathrm{B}_{1} \& \mathrm{~B}_{2} \& \ldots \& \mathrm{~B}_{\mathrm{m}-1} \& \mathrm{~B}_{\mathrm{m}} \\
& \quad \ldots \\
& \# \sim \mathrm{A}_{1} \& \sim \mathrm{A}_{2} \& \ldots \& \sim \mathrm{A}_{\mathrm{m}-1} \& \mathrm{~A}_{\mathrm{m}}=\# \sim \mathrm{B}_{1} \& \sim \mathrm{B}_{2} \& \ldots \& \sim \mathrm{B}_{\mathrm{m}-1} \& \mathrm{~B}_{\mathrm{m}} \\
& \# \sim \mathrm{A}_{1} \& \sim \mathrm{A}_{2} \& \ldots \& \sim \mathrm{A}_{\mathrm{m}-1} \& \sim \mathrm{A}_{\mathrm{m}}=\# \sim \mathrm{B}_{1} \& \sim \mathrm{B}_{2} \& \ldots \& \sim \mathrm{B}_{\mathrm{m}-1} \& \sim \mathrm{B}_{\mathrm{m}}
\end{aligned}
$$

where there is one equality for each of the $2^{\mathrm{m}}$ combinations of unnegated and negated propositions in the list of $\mathrm{m}$ propositions.

To see this, note that if $\mathrm{A}_{1} \& \mathrm{~A}_{2} \& \ldots \& \mathrm{~A}_{\mathrm{m}-1} \& \mathrm{~A}_{\mathrm{m}}$ and $\mathrm{B}_{1} \& \mathrm{~B}_{2} \& \ldots \& \mathrm{~B}_{\mathrm{m}-1} \& \mathrm{~B}_{\mathrm{m}}$ are disjunctions of the same number of atoms, the atoms in $A_{1}, \ldots, A_{m}$ can be relabeled so that they match the labels of $\mathrm{B}_{1} \& \mathrm{~B}_{2} \& \ldots \& \mathrm{~B}_{\mathrm{m}-1} \& \mathrm{~B}_{\mathrm{m}}$; and so on for the remaining $2^{\mathrm{m}_{-}}-1$ conjunctions. Since the $2^{\mathrm{m}}$ conjunctions are exclusive, none of these mapping will conflict; and since the $2^{\mathrm{m}}$ conjunctions exhaust the algebra, the relabeling is a complete mapping of all the atoms back onto themselves. ${ }^{13}$

\section{B. Proof of the No-Go Result}

\section{B.1 Classes of Deductive Structures}

Take the fixed set of propositions $\left\{A_{1}, \ldots, A_{m}\right\}$ of the explicit or implicit definition of a deductively definable logic of induction. We investigate how the strengths $\left[A_{i} \mid A_{k}\right]$ vary under arbitrary disjunctive refinement of the algebra. To track these variations, we categorize the

13 A quick way to see these last claims is to draw a Venn diagram of an example and note that the $2^{\mathrm{m}}$ conjunctions correspond to the $2^{\mathrm{m}}$ smallest areas in the diagram. 
strengths according to the deductive structure of the propositions $\left\{\mathrm{A}_{1}, \ldots, \mathrm{A}_{\mathrm{m}}\right\}$; that is, according to the full list of which propositions entail which propositions, which contradict which and which are logically compatible with which. That is:

Classes of Deductive Structures. Strengths $\left[\mathrm{A}_{\mathrm{i}} \mid \mathrm{A}_{\mathrm{k}}\right]$ and $\left[\mathrm{B}_{\mathrm{r}} \mid \mathrm{B}_{\mathrm{S}}\right]$ are drawn from the same class of deductive structures ${ }^{14}$ among propositions $\left\{A_{1}, \ldots, A_{m}\right\}$ and $\left\{B_{1}, \ldots\right.$, $B_{m}$ \} respectively just in case the logical relations among the $A_{i}$ and $A_{k}$ and the remaining propositions in the set are the same as the logical relations among the $\mathrm{B}_{\mathrm{r}}$ and $\mathrm{B}_{\mathrm{s}}$ and the remaining propositions in the set.

A convenient way of distinguishing the classes is to identify which of the conjunctions in the list of $2^{\mathrm{m}}$ conjunctions in the symmetry theorem above are $\varnothing$ and thus have zero atoms.

In the simplest case of a two proposition list, A, B, the classes include: A entails B (for which $\mathrm{A} \& \sim \mathrm{B}=\varnothing$ ); $\mathrm{B}$ entails $\mathrm{A}$ (for which $\sim \mathrm{A} \& \mathrm{~B}=\varnothing$ ); $\mathrm{A}$ and $\mathrm{B}$ are logically incompatible (for which $\mathrm{A} \& \mathrm{~B}=\varnothing$ ); $\mathrm{A}$ and $\mathrm{B}$ are logically compatible and disjunctively exhaust the algebra (for which $\sim \mathrm{A} \& \sim \mathrm{B}=\varnothing$ ); $\mathrm{A}$ and $\mathrm{B}$ are logically compatible but do not disjunctively exhaust the algebra (for which no conjunctions are $\varnothing$ ).

Thus membership of two sets of propositions in the same class is revealed when, under suitable relabeling of one of the sets, the two functions f defining the inductive strengths have zero values for corresponding arguments.

\section{B.2 Dynamics under Disjunctive Refinement}

We now show that the strengths $[\mathrm{A} / \mathrm{B}]$ and $[\mathrm{CID}]$ must converge to the same limiting strengths if they are drawn from the same class of deductive structures. Consider the $\mathrm{m}(\mathrm{m}-1)$ non-trivial ${ }^{15}$ strengths that can be defined using the propositions in the set $\left\{A_{1}, \ldots, A_{m}\right\}$ :

14 This amount of deductive structure is less than the full deductive structure invoked in the definitions of deductively definable logics of induction, for the latter definitions include deductive relations with the atoms of the algebra.

15 That is, we exclude $\left[\mathrm{A}_{1} \mid \mathrm{A}_{1}\right],\left[\mathrm{A}_{2} \mid \mathrm{A}_{2}\right], \ldots$, which are equal by supposition. 
$\left[A_{1} \mid A_{2}\right],\left[A_{1} \mid A_{3}\right], \ldots,\left[A_{m-1} \mid A_{m}\right]$. If there are two strengths drawn from the same class of deductive structures here, then there are two relabelings of the propositions $A_{1}, \ldots, A_{m}$ such that the first strength is labeled $\left[A_{1} \mid A_{2}\right]$ and the remaining propositions $A_{3}, \ldots, A_{m}$; and the second strength is labeled $\left[\mathrm{B}_{1} \mid \mathrm{B}_{2}\right]$ and the remaining propositions $\mathrm{B}_{3}, \ldots, \mathrm{B}_{\mathrm{m}}$. Moreover, the relabelings have the following property.

Consider the conjunctions of propositions mentioned in the symmetry theorem. The distribution of null $\varnothing$ among them encodes the class of deductive structure. For example if $A_{2}$, $\ldots \mathrm{A}_{\mathrm{m}}$ entails $\mathrm{A}_{1}$, then $\sim \mathrm{A}_{1} \& \mathrm{~A}_{2} \& \ldots \& \mathrm{~A}_{\mathrm{m}}=\varnothing$. Therefore, correspondingly, we must have $\mathrm{B}_{2}$, $\ldots, \mathrm{B}_{\mathrm{m}}$ entails $\mathrm{B}_{1}$, so that $\sim \mathrm{B}_{1} \& \mathrm{~B}_{2} \& \ldots \& \mathrm{~B}_{\mathrm{m}}=\varnothing$. Hence the corresponding conjunctions of propositions will agree on which are the null $\varnothing$ members. That is

$$
\begin{aligned}
& \mathrm{A}_{1} \& \mathrm{~A}_{2} \& \ldots \& \mathrm{~A}_{\mathrm{m}-1} \& \mathrm{~A}_{\mathrm{m}}=\varnothing \quad \text { iff } \quad \mathrm{B}_{1} \& \mathrm{~B}_{2} \& \ldots \& \mathrm{~B}_{\mathrm{m}-1} \& \mathrm{~B}_{\mathrm{m}}=\varnothing \\
& \sim \mathrm{A}_{1} \& \mathrm{~A}_{2} \& \ldots \& \mathrm{~A}_{\mathrm{m}-1} \& \mathrm{~A}_{\mathrm{m}}=\varnothing \quad \text { iff } \quad \sim \mathrm{B}_{1} \& \mathrm{~B}_{2} \& \ldots \& \mathrm{~B}_{\mathrm{m}-1} \& \mathrm{~B}_{\mathrm{m}}=\varnothing \\
& \text { etc. }
\end{aligned}
$$

It now follows that corresponding arguments in the function $f$ for $\left[\mathrm{A}_{1} \mid \mathrm{A}_{2}\right]$ and $\left[\mathrm{B}_{1} \mid \mathrm{B}_{2}\right]$ agree in those conjunctions that have zero atoms counts and those which have non-zero atom counts.

Using this key fact, the evolution of these two strengths under disjunctive refinement is tracked in two stages:

Stage 1. The algebra is disjunctively refined until the atom counts of the conjunctions of the propositions $A_{i}$ in the refined algebra equal or exceed the corresponding atom counts in conjunctions of the propositions $B_{i}$ in the unrefined algebra:

$$
\begin{aligned}
& \left(\# \mathrm{~A}_{1} \& \mathrm{~A}_{2} \& \ldots \& \mathrm{~A}_{\mathrm{m}-1} \& \mathrm{~A}_{\mathrm{m}}\right)_{\text {refined }} \geq\left(\# \mathrm{~B}_{1} \& \mathrm{~B}_{2} \& \ldots \& \mathrm{~B}_{\mathrm{m}-1} \& \mathrm{~B}_{\mathrm{m}}\right)_{\text {unrefined }} \\
& \ldots \\
& \left.\left(\# \sim \mathrm{A}_{1} \& \sim \mathrm{A}_{2} \& \ldots \& \sim \mathrm{A}_{\mathrm{m}-1} \& \sim \mathrm{A}_{\mathrm{m}}\right)_{\text {refined }} \geq \# \sim \mathrm{B}_{1} \& \sim \mathrm{B}_{2} \& \ldots \& \sim \mathrm{B}_{\mathrm{m}-1} \& \sim \mathrm{B}_{\mathrm{m}}\right)_{\text {unrefined }}
\end{aligned}
$$

It is now possible to carry out a different refinement of the original algebra (labeled below as "refined*") under which the above inequalities become equalities:

$$
\begin{aligned}
& \left(\# \mathrm{~A}_{1} \& \mathrm{~A}_{2} \& \ldots \& \mathrm{~A}_{\mathrm{m}-1} \& \mathrm{~A}_{\mathrm{m}}\right)_{\text {refined }}=\left(\# \mathrm{~B}_{1} \& \mathrm{~B}_{2} \& \ldots \& \mathrm{~B}_{\mathrm{m}-1} \& \mathrm{~B}_{\mathrm{m}}\right)_{\text {refined }} * \\
& \ldots \\
& \left(\# \sim \mathrm{A}_{1} \& \sim \mathrm{A}_{2} \& \ldots \& \sim \mathrm{A}_{\mathrm{m}-1} \& \sim \mathrm{A}_{\mathrm{m}}\right)_{\text {refined }}=\left(\# \sim \mathrm{B}_{1} \& \sim \mathrm{B}_{2} \& \ldots \& \sim \mathrm{B}_{\mathrm{m}-1} \& \sim \mathrm{B}_{\mathrm{m}}\right)_{\text {refined }} *
\end{aligned}
$$

It follows from the symmetry theorem of Section 4 that these two strengths are equal: 


$$
\left[\mathrm{A}_{1} \mid \mathrm{A}_{2}\right]_{\text {refined }}=\left[\mathrm{B}_{1} \mid \mathrm{B}_{2}\right]_{\text {refined* }}
$$

Stage 2. We now refine the algebra as extensively as is needed to drive the strength $\left[\mathrm{A}_{1} \mid \mathrm{A}_{2}\right]$ towards its unique limiting value $\left[\mathrm{A}_{1} \mid \mathrm{A}_{2}\right]_{\mathrm{lim}}$. The assumption of asymptotic stability assures us that there is such a unique limit and that any strength $\left[\mathrm{A}_{1} \mid \mathrm{A}_{2}\right]$ can be driven to converge to it by suitable selection of disjunctive refinements.

We also carry out the corresponding refinements* in association with the strength $\left[\mathrm{B}_{1} \mid \mathrm{B}_{2}\right]$ so that the equalities of the atom counts of the conjunctions continue to hold. Hence the strength $\left[\mathrm{B}_{1} \mid \mathrm{B}_{2}\right]$ is driven towards its unique limiting value $\left[\mathrm{B}_{1} \mid \mathrm{B}_{2}\right]_{\text {lim }}$. By the symmetry theorem of Section 4, these two limits must agree:

$$
\left[\mathrm{A}_{1} \mid \mathrm{A}_{2}\right]_{\text {lim }}=\left[\mathrm{B}_{1} \mid \mathrm{B}_{2}\right]_{\text {lim }}
$$

Thus we have found that sufficient disjunctive refinement drives all inductive strengths associated with the same class of deductive structures towards the same limiting value, which may or may not be unique to that class.

\section{B.3 Continuity}

The continuity condition for a deductively definable logic of induction is expressed in terms of the function $\mathrm{f}$. Since $\mathrm{f}$ is not a function on the real numbers, but a function of natural numbers, the familiar notion of continuity for functions on the real numbers does not apply. A weaker notion is definable. Loosely speaking, it asserts that, for very large atom counts, differences in the absolute numbers of the atom counts become unimportant to the strengths and their effects can be made arbitrarily small. More precisely:

Continuity in one argument. The function $\mathrm{f}$ of the symmetry theorem is continuous in the argument $X$ : if $V(X)=f(\ldots, u, v, X, y, z, \ldots)$ and $V\left(X^{\prime}\right)=f\left(\ldots, u, v, X^{\prime}, y\right.$, $\mathrm{z}, \ldots)$, then $\mathrm{V}(\mathrm{X})$ approaches $\mathrm{V}\left(\mathrm{X}^{\prime}\right)$ in the limit in which $\mathrm{X}$ and $\mathrm{X}^{\prime}$ are held fixed and $\ldots, \mathrm{u}, \mathrm{v}, \mathrm{y}, \mathrm{z}, \ldots$ each grow arbitrarily large, unless any of the $\ldots, \mathrm{u}, \mathrm{v}, \mathrm{y}, \mathrm{z}, \ldots$ are 0 , in which case they remain 0.16

Continuity. The function $f$ is continuous, if it is continuous in each of its arguments.

16 If all the $\ldots, \mathrm{u}, \mathrm{v}, \mathrm{y}, \mathrm{z}, \ldots$ are 0 , we have a degenerate case of no interest in inductive logic. The condition of continuity places no restriction on the strengths. 
The notion of limit in this definition is an obvious one: for any strengths $V^{\prime}<V^{\prime}$, , there exists variable values $\ldots, u^{*}, v^{*}, y^{*}, z^{*}, \ldots$ such that for all $\ldots, u^{\prime}>u^{*}, v^{\prime}>v^{*}, y^{\prime}>y^{*}, z^{\prime}>z^{*}, \ldots$

$$
V^{\prime}<V(X)=f\left(\ldots, u^{\prime}, v^{\prime}, X, y^{\prime}, z^{\prime}, \ldots\right)<V^{\prime},
$$

just in case we also have

$$
V^{\prime}<V\left(X^{\prime}\right)=f\left(\ldots, u^{\prime}, v^{\prime}, X^{\prime}, y^{\prime}, z^{\prime}, \ldots\right)<V^{\prime},
$$

This notion of continuity is satisfied by typical functions $\mathrm{f}$. In particular, it is satisfied by any function $f$ whose value depends only on the ratios of the atoms counts, such as the $f$ associated with classical probability: $[\mathrm{A} \mid \mathrm{B}]=\# \mathrm{~A} \& \mathrm{~B} / \# \mathrm{~B}$.

\section{B.4 Reduction to a Single Strength}

The analysis of the Section B.2 shows that the limiting values of the inductive strengths converge to a finite set, with one limit for each class of deductive structures of the set of propositions $\left\{\mathrm{A}_{1}, \ldots, \mathrm{A}_{\mathrm{m}}\right\}$. It will follow from the continuity condition that all these limits are the same.

The classes of deductive structures as a whole form a larger structure in which we can move step-wise from one class to another adjacent to it merely by setting one of the conjunctions in the symmetry theorem to $\varnothing$, or, conversely, unsetting it. The easiest entry point is the deductive structure in which none of the $2^{\mathrm{m}}$ conjunctions $\mathrm{A}_{1} \& \mathrm{~A}_{2} \& \ldots \& \mathrm{~A}_{\mathrm{m}-1} \& \mathrm{~A}_{\mathrm{m}}, \ldots$, $\sim \mathrm{A}_{1} \& \sim \mathrm{A}_{2} \& \ldots \& \sim \mathrm{A}_{\mathrm{m}-1} \& \sim \mathrm{A}_{\mathrm{m}}$ are $\varnothing$. This corresponds to each of the $\mathrm{A}_{1}, \ldots, \mathrm{A}_{\mathrm{m}}$ being logically compatible with each other but not disjunctively exhaustive of the algebra. We then move to the other classes by setting repeatedly one or other of conjunctions to $\varnothing$.

For the case of $\{\mathrm{A}, \mathrm{B}, \mathrm{C}\}$, we start with none of the $2^{3}=8$ conjunctions $\mathrm{A} \& \mathrm{~B} \& \mathrm{C}, \ldots$, $\sim \mathrm{A} \& \sim \mathrm{B} \& \sim \mathrm{C}$ being $\varnothing$ :

(a) If we set $\sim \mathrm{A} \& \sim \mathrm{B} \& \sim \mathrm{C}=\varnothing$, then the propositions $\mathrm{A}, \mathrm{B}$ and $\mathrm{C}$ are disjunctively exhaustive: $\mathrm{AvBvC}=\Omega$.

(b) If we set $\mathrm{A} \& \sim \mathrm{B} \& \mathrm{C}=\varnothing$ and $\mathrm{A} \& \sim \mathrm{B} \& \sim \mathrm{C}=\varnothing$, then $\mathrm{A} \& \sim \mathrm{B}=\varnothing$ and $\mathrm{A}$ deductively entails B.

(c) If in addition to (b) we set $\sim \mathrm{A} \& \mathrm{~B} \& \mathrm{C}=\varnothing$ and $\sim \mathrm{A} \& \mathrm{~B} \& \sim \mathrm{C}=\varnothing$, then $\sim \mathrm{A} \& \mathrm{~B}=\varnothing$ and $\mathrm{B}$ also deductively entails $\mathrm{A}$. In this combined case, $[\mathrm{A} \mid \mathrm{B}]=[\mathrm{A} \mid \mathrm{A}]=$ $[\Omega \mid \Omega]$, the maximum strength. 
(d) If we set $\mathrm{A} \& \mathrm{~B} \& \mathrm{C}=\varnothing$ and $\mathrm{A} \& \mathrm{~B} \& \sim \mathrm{C}=\varnothing$, then $\mathrm{A} \& \mathrm{~B}=\varnothing$ and $\mathrm{B}$ contradicts $\mathrm{A}$. In this case, $[\mathrm{A} \mid \mathrm{B}]=[\mathrm{B} \mid \mathrm{A}]=[\varnothing \mid \Omega]$, the minimum strength.

In this way, by sequentially setting one or other conjunction to $\varnothing$, we can visit all the deductive classes to which the strengths can belong, including the cases of maximum and minimum strength.

Consider two strengths $\left[\mathrm{A}_{\mathrm{i}} \mid \mathrm{A}_{\mathrm{k}}\right]$ in $\left\{\mathrm{A}_{1}, \ldots, \mathrm{A}_{\mathrm{m}}\right\}$ and $\left[\mathrm{B}_{\mathrm{r}} \mathrm{I} \mathrm{B}_{\mathrm{s}}\right]$ in $\left\{\mathrm{B}_{1}, \ldots, \mathrm{B}_{\mathrm{m}}\right\}$. We shall say that they are drawn from adjacent classes if we can move one strength from its class to the other class merely by setting one of its non-null conjunctions to $\varnothing$. For concreteness, let us say in this case that, when we set one conjunction from the A-class to $\varnothing$, we move the strength $\left[\mathrm{A}_{\mathrm{i}} \mid \mathrm{A}_{\mathrm{k}}\right]$ to the adjacent $\mathrm{B}$-class as $\left[\mathrm{B}_{\mathrm{r}} \mid \mathrm{B}_{\mathrm{S}}\right]$. That is, we can write the two strengths as:

$$
\begin{aligned}
& {\left[A_{i} \mid A_{k}\right]=f(\ldots, u, v, x, y, z, \ldots)} \\
& {\left[B_{r} \mid B_{s}\right]=f(\ldots, u, v, 0, y, z, \ldots)}
\end{aligned}
$$

where $\mathrm{x}>0$ and the $\ldots, \mathrm{u}, \mathrm{v}, \mathrm{y}, \mathrm{z}, \ldots$ are now limited to just those atom counts not already set to zero in the first class of deductive structures.

To see that these two strengths converge to the same limiting values under sufficient disjunctive refinement, pick any small interval of values bounded by $\mathrm{V}<\mathrm{V}$ '. Because the logic is assumed continuous in the sense of the last section, there exists variable values $\ldots, \mathrm{u}^{*}, \mathrm{v}^{*}, \mathrm{y}^{*}, \mathrm{z}^{*}$, $\ldots$ such that for all $\ldots, u^{\prime}>u^{*}, v^{\prime}>v^{*}, y^{\prime}>y^{*}, z^{\prime}>z^{*}, \ldots$

$$
\mathrm{V}<\left[\mathrm{A}_{\mathrm{i}} \mid \mathrm{A}_{\mathrm{k}}\right]=\mathrm{f}\left(\ldots, \mathrm{u}^{\prime}, \mathrm{v}^{\prime}, \mathrm{X}, \mathrm{y}^{\prime}, \mathrm{z}^{\prime}, \ldots\right)<\mathrm{V}^{\prime}
$$

just in case we also have

$$
\mathrm{V}<\left[\mathrm{B}_{\mathrm{r}} \mid \mathrm{B}_{\mathrm{s}}\right]=\mathrm{f}\left(\ldots, \mathrm{u}^{\prime}, \mathrm{v}^{\prime}, \mathrm{X}^{\prime}, \mathrm{y}^{\prime}, \mathrm{z}^{\prime}, \ldots\right)<\mathrm{V}^{\prime}
$$

Since the interval bounded by $\mathrm{V}<\mathrm{V}^{\prime}$ is arbitrary, it can be made arbitrarily small. It follows that $\left[\mathrm{A}_{\mathrm{i}} \mid \mathrm{A}_{\mathrm{k}}\right]$ and $\left[\mathrm{B}_{\mathrm{r}} \mid \mathrm{B}_{\mathrm{S}}\right]$ approach the same limiting value under continuing disjunctive refinement:

$$
\left[\mathrm{A}_{\mathrm{i}} \mid \mathrm{A}_{\mathrm{k}}\right]_{\lim }=\left[\mathrm{B}_{\mathrm{r}} \mid \mathrm{B}_{\mathrm{s}}\right]_{\lim }
$$

We have from Section B.2 that all strengths in one class of deductive structures converge to the same limit under disjunctive refinement. Therefore this last common limiting strength is the limit strength for the two classes from which $\left[\mathrm{A}_{\mathrm{i}} \mid \mathrm{A}_{\mathrm{k}}\right]$ and $\left[\mathrm{B}_{\mathrm{r}} \mid \mathrm{B}_{\mathrm{S}}\right]$ are drawn.

Each of $\left[A_{i} \mid A_{k}\right]$ and $\left[B_{r} \mid B_{s}\right]$ are chosen generally with the sole restriction that they are drawn from adjacent classes of deductive structures. Therefore we have the general result that 
the limiting strengths for any pair of adjacent classes are the same. Finally, all the classes are connected with one another by a chain of classes, each of which is adjacent to the next.

Therefore the limiting strengths for all classes are the same. This includes the cases in which the limiting strengths are the maximum $[\Omega \mid \Omega]$ and the minimum $[\varnothing \mid \Omega]$.

\section{References}

Finetti, Bruno de. 1938. "Sur la condition d'equivalence partielle." Actualités Scientifique et Industrielles, No. 739, Hermann \& Cie., Paris. Translated as "On the Condition of Partial Exchangeability," Ch. 9 in Jeffrey, Richard C., ed., Studies in Inductive Logic and Probability, Vol. 2, Univ. of California Press, 1980.

Fitelson, Branden. 1999. "The Plurality of Bayesian Measures of Confirmation and the Problem of Measure Sensitivity." Philosophy of Science 66: S362-78.

Gandenberger, Greg. manuscript. "Why I Am Not a Methodological Likelihoodist." http://gandenberger.org/wp-content/uploads/2014/10/wianaml23_4.pdf

Hempel, Carl G. 1965. "Studies in the Logic of Confirmation." Ch.1 in Aspects Of Scientific Explanation And Other Essays In The Philosophy Of Science. New York: Free Press, 1965.

Kelly, Kevin T. 1996. The Logic of Reliable Inquiry. New York: Oxford University Press. Keynes, John Maynard. 1921. A Treatise on Probability. London: MacMillan.

Norton, John D. 2003. "A Material Theory of Induction." Philosophy of Science 70: 647-70. Norton, John D. 2008. "Ignorance and Indifference." Philosophy of Science 75: 45-68.

Norton, John D. 2010. "Deductively Definable Logics of Induction." Journal of Philosophical Logic. 39: 617-654.

Norton, John D. 2015. “The Ideal of the Completeness of Calculi of Inductive Inference: An Introductory Guide to its Failure.” http://philsci-archive.pitt.edu/11937/

Putnam, Hilary. 1963 “'Degree of Corroboration' and Inductive Logic,” Ch. 17 in H. Putnam, Mathematical Matter and Method: Philosophical Papers Volume 1.2nd ed. Cambridge: Cambridge University Press, 1979.

Savage, Leonard J. 1954. The Foundations of Statistics. John Wiley \& Sons; reprinted New York: Dover, 1972. 
Shimony, Abner. 1970. "Scientific Inference," pp. 79-172 in R. G. Colodny, ed., The Nature and Function of Scientific Theories: University of Pittsburgh Series in the Philosophy of Science, Vol. 4. Pittsburgh: University of Pittsburgh Press.

Solomonoff, Ray. 1964. "A Formal Theory of Inductive Inference." Information and Control 7: $1-22 ; 224-254$.

Titelbaum, Michael. 2010. "Not Enough There There: Evidence, Reasons, and Language Independence." Philosophical Perspectives. 24: 477-528.

Titelbaum, Michael. 2011. "Symmetry and Evidential Support.” Symmetry 3: 680-698. 